\title{
Applications of Shock-Induced Mixing to Supersonic Combustion
}

\author{
Joseph Yang,* Toshi Kubota, $†$ and Edward E. Zukoskił \\ California Institute of Technology, Pasadena, California 91125
}

\begin{abstract}
Families of two-dimensional, unsteady shock-induced vortical flows are simulated numerically. The flows consist of one or more regions of light gas, surrounded by heavy gas, being overtaken by a normal shock wave. The interaction of the density gradient at each light/heavy interface with the pressure gradient from the shock wave generates vorticity. This causes the light gas regions to roll up into one or more counter-rotating vortex pairs, which stir and mix the light and heavy gases. The mixing is characterized by an asymptotic stretching rate. The effects of shock strength, light/heavy gas density ratio, and geometry on the mixing are investigated. These two-dimensional, unsteady flows are analogous to three-dimensional, steady flows that may be used in SCRAMJET combustors demanding rapid and efficient mixing of fuel and oxidizer. For such applications, 1) the fuel injectors should be elongated in the direction of the shock; 2) multiple smaller injectors are preferable to a single larger injector; 3 ) injectors should be arranged in groups of closely spaced pairs, rather than uniformly; and 4) multiple shock waves should be utilized, if possible.
\end{abstract}

\section{Introduction}

$\mathbf{R}$ APID and efficient mixing of fuel and oxidizer is crucial for supersonic combustion ramjet (SCRAMJET) propulsion, where the combustion chamber residence time will be on the order of milliseconds. One likely strategy is shock-induced mixing, which was first proposed by Marble ${ }^{1}$ in 1985, based on two key insights. First, he recognized that two-dimensional shock scattering by cylinders of low density gas, investigated by Haas $^{2}$ and Haas and Sturtevant, ${ }^{3}$ suggested a mechanism of greatly enhancing the rate of mixing between the light and heavy gases. Second, he saw that the two-dimensional, unsteady flow was a good analog for the three-dimensional, steady flow produced when an oblique shock impinges on a light gas jet immersed in a coflowing and supersonic air stream.

The two-dimensional, unsteady flow is more convenient to study, either experimentally or numerically, than the three-dimensional, steady flow. One may define a canonical shock-induced vortical flow (Fig. 1) as the two-dimensional, unsteady passage of a normal shock wave over a single circular region of light gas (e.g., a cross section through a jet) surrounded by ambient heavy gas. Misalignment of the density gradient at the edge of the jet with the pressure gradient due to the normal shock generates vorticity. This rolls up the jet into a counterrotating vortex pair.

The dynamics of this flow have been the subject of numerous investigations ${ }^{2-8}$ and will not be discussed here. The present work considers the kinematics of the flow, in particular the mixing and its dependence on shock strength and light/heavy gas density ratio. The mixing is characterized as an asymptotic stretching rate determined from numerical simulations.

The canonical flow conveniently illustrates the basic phenomenon and provides a baseline against which the mixing effectiveness of more complicated flows can be compared. Flows of practical interest are likely to consist of one or more jets of noncircular cross section. Thus, families of flows involving variations in single jet cross-sectional shape and

Received Oct. 2, 1991; revision received Sept. 28, 1992; accepted for publication Oct. 8, 1992. Copyright (C) 1992 by the American Institute of Aeronautics and Astronautics, Inc. All rights reserved.

*Graduate Research Assistant; currently Associate Research Engineer, Shell Development Company, P. O. Box 1380, Houston, TX 77251. Member AIAA.

$\dagger$ Professor Emeritus of Aeronautics, 301-46 Caltech. Member AIAA.

$\ddagger$ Professor of Jet Propulsion and Mechanical Engineering, 301-46 Caltech. Fellow AIAA. multiple jet array geometry are simulated. Flows involving interactions with multiple shocks are also considered.

The mixing implications from these two-dimensional, unsteady flows are interpreted in terms of their corresponding three-dimensional, steady counterparts. This establishes guidelines for selection of fluid mechanical and geometrical parameters to enhance the mixing during fuel injection in a SCRAMJET.

\section{Computational Technique}

The flow is governed by the usual conservation equations for continuity, momentum, energy, and species for two-dimensional, unsteady flow. Physical variables are nondimensionalized according to the following conventions:

1) Scale lengths by the initial radius: $\bar{x}=x / R_{0}$.

2) Scale velocities by the speed of sound ahead of the shock: $\bar{u}=u / c_{1}$.

3) Scale times by $R_{0} / c_{1}: t=t c_{1} / R_{0}$.

4) Scale densities by the density ahead of the shock: $\bar{\rho}=$ $\rho / \rho_{1}$

5) Scale pressures by the pressure ahead of the shock: $\bar{p}=p / p_{1}$

The governing equations are solved using LCPFCT, ${ }^{9}$ the most recent version of an explicit, Eulerian, finite-difference flux-corrected transport (FCT) algorithm developed by Boris. ${ }^{10}$ The usual difficulty in finite-difference computations is that the numerical diffusion required for stability often overwhelms the actual physical diffusion. Thus it is difficult to capture strong gradients, such as shock waves, without smearing out fine details of the flow. LCPFCT features an antidiffusive correction stage that locally removes the numerical diffusion in excess of either the physically correct diffusion or that required for stability. In the computations discussed here,

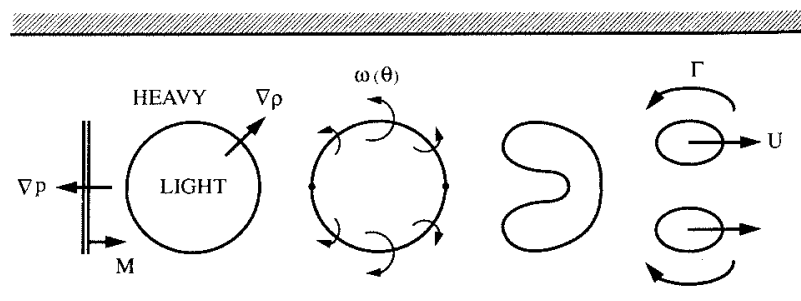

Fig. 1 Canonical shock-induced vortical flow: preshock, postshock, roll up, and steady state. 
approximately $0.1 \%$ of the original numerical diffusion is retained to match the physical diffusion.

The flow is initialized by specifying density, velocity, pressure, and concentration fields for the jet, the ambient fluid surrounding the jet, and the normal shock wave impinging from left to right. The jet is initialized using a similarity solution for the mixing layer at the edge of an axisymmetric jet, at a plane 4.8 diameters downstream of the nozzle.

The initial condition is integrated forward in time, with a time step determined by a Courant condition. The $x$ and $y$ integrations are treated separately, using the technique of operator splitting. The grid size is 0.05 square $(5 \%$ of the radius of the jet, which is defined to be unity); additional computations at grid size 0.025 confirmed the resolution adequacy of the 0.05 grid spacing. During the computation, the domain is moved in time to track the developing vortex pair. The inflow and outflow boundaries specify zero-gradient flow. The upper and lower boundaries are perfectly reflecting; this corresponds physically to jets symmetrically located about the centerline of a channel of finite height.

\section{Characterization of the Mixing}

Mixing is a two-step process. The first step, stretching, is a necessary precursor to the second step, diffusion, because it is through gradient intensification that diffusive effects become important. This suggests that an appropriately defined stretching rate can be used as a characterization of the mixing.

Batchelor ${ }^{11}$ investigated the effect of homogeneous turbulence on material elements of fluid and found the best achievable stretching rate to be exponential. More recently, other investigators have found that exponential stretching is also possible, at least locally, in essentially inviscid two-dimensional, unsteady or three-dimensional, steady flows. ${ }^{12}$ In expectation of this possibility, the specific stretching rate

$$
\frac{1}{\bar{L}} \frac{\mathrm{D} \bar{L}}{\mathrm{D} \bar{t}}=\frac{\mathrm{D}}{\mathrm{D} \bar{t}}[\ln \bar{L}]
$$

should asymptotically approach a constant value as a function of time.

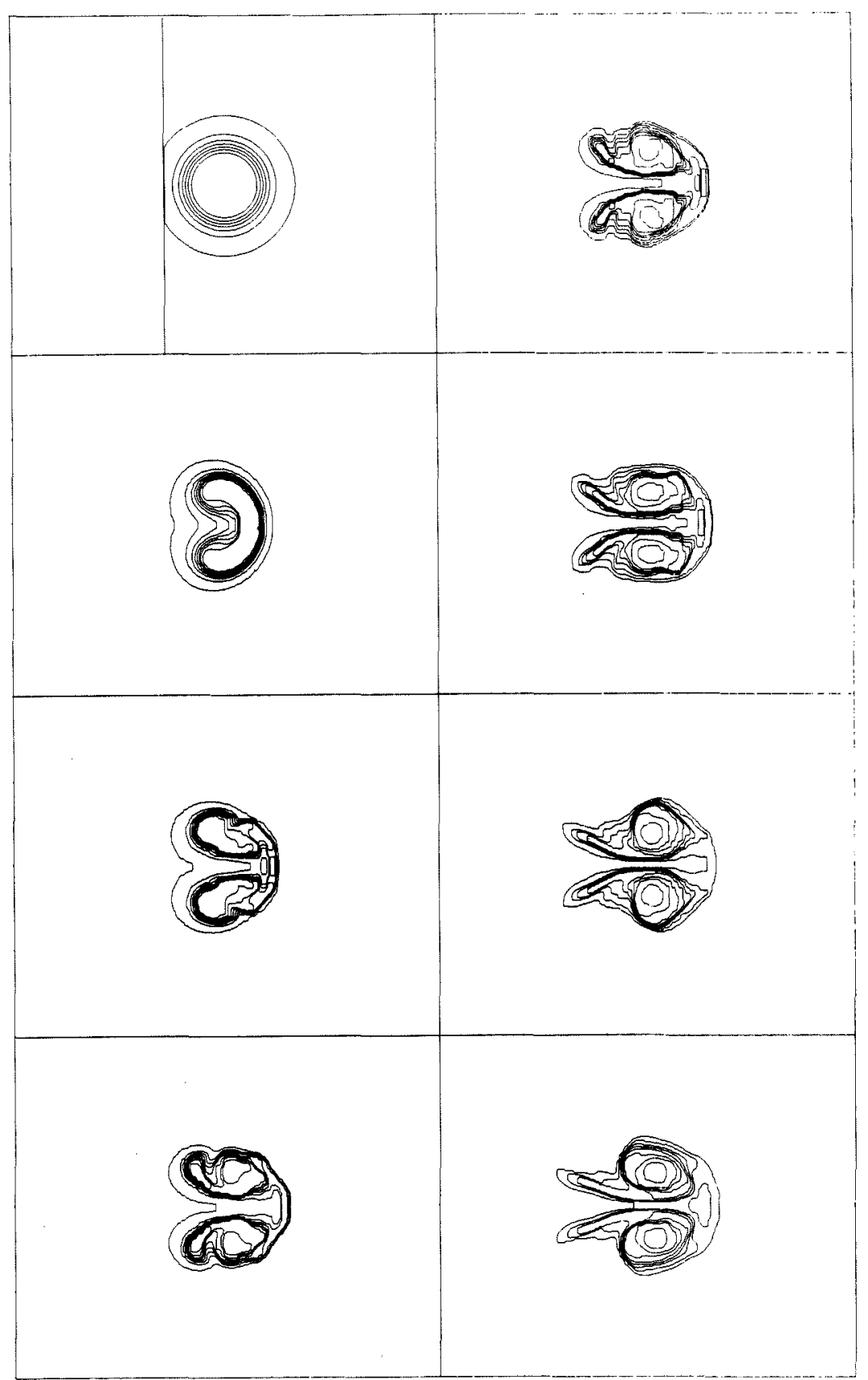

Fig. 2 Density contours at $\bar{t}=0-70$ for a single circular jet with $M=1.1$ and $\bar{\rho}_{L} / \bar{\rho}_{H}=0.138$. 


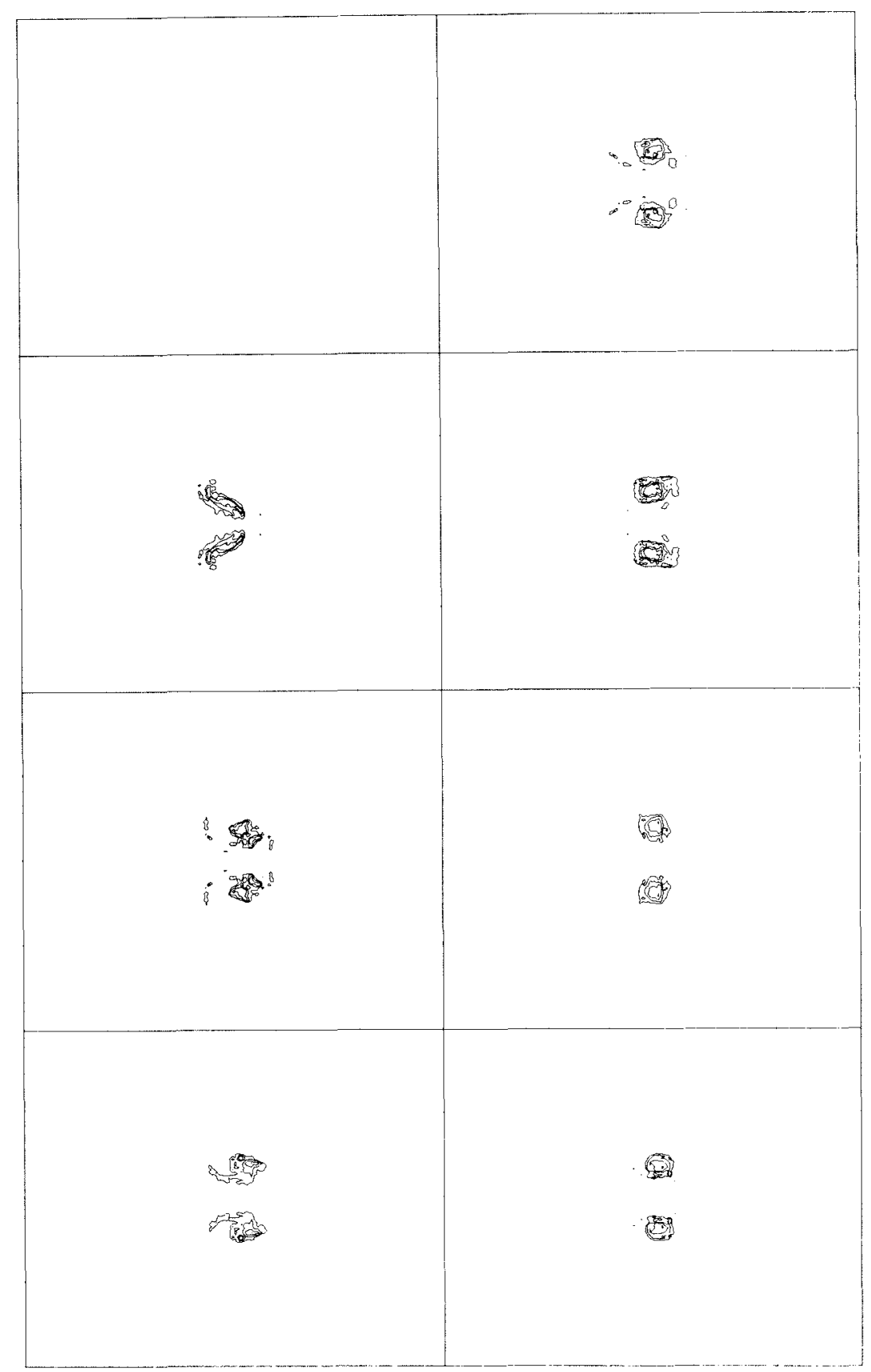

Fig. 3 Vorticity contours at $\ddot{t}=0-70$ for a single circular jet with $M=1.1$ and $\bar{\rho}_{L} / \bar{\rho}_{H}=0.138$.

$\bar{L}$ is the length of a material element following the fluid, corresponding to a particular mass-fraction level of interest. For combustion of hydrogen in air, the balanced chemical equation is

$$
2 \mathrm{H}_{2}+\left(3.76 \mathrm{~N}_{2}+\mathrm{O}_{2}\right) \rightarrow 3.76 \mathrm{~N}_{2}+2 \mathrm{H}_{2} \mathrm{O}
$$

and the stoichiometric fuel mass fraction is $f_{s}=0.028$. The anticipated technological application for shock-induced mixing is a SCRAMJET, which must carry excess hydrogen for cooling the structure in flight. This suggests that the actual fuel mass fraction will be on the order of $f \approx 0.05$.

Lengths of the $f=0.05$ contours are determined numerically from contour plots of mass fraction, at various times throughout the flow. Initially, the stretching rate increases monotonically. At late times, it drops off as either diffusive effects become important or some of the fluid enclosed by the contour of interest is stretched thinner than the cell size and can no longer be resolved. In either case, the dropoff should correspond to the analogous point in a real flow, but the quantitative reliability of the data beyond that point is not guaranteed. Thus, as a mixing measure, one defines a critical stretching rate as that achieved at the drop-off point. This represents the highest stretching that is achieved in the flow before resolution or diffusion losses become important, and provides a basis for comparing the mixing effectiveness of the various families of flows.

\section{Single Circular Jet}

Consider the canonical flow of a single circular jet being overtaken by a normal shock (Fig. 1). Vorticity is generated baroclinically according to the inviscid vorticity equation

$$
\frac{\mathrm{D}}{\mathrm{D} t}\left(\frac{\omega}{\rho}\right)=\frac{1}{\rho}(\omega \cdot \nabla u)+\frac{1}{\rho^{3}}(\nabla \rho \times \nabla p)
$$


Table 1 Stretching rates

\begin{tabular}{|c|c|c|c|c|c|}
\hline No. & Description & $M$ & $\bar{\rho}_{L} / \bar{\rho}_{H}$ & $\frac{\mathrm{D}}{\mathrm{D} \bar{t}}[\ln \bar{L}(f=0.05)]$ & $\begin{array}{l}\text { Change from corrected } \\
\text { canonical flow, } \%\end{array}$ \\
\hline 1 & Canonical flow & 1.05 & 0.138 & 0.007 & - \\
\hline 2 & Canonical flow & 1.1 & 0.138 & 0.014 & - \\
\hline 3 & Canonical flow & 1.2 & 0.138 & 0.024 & - \\
\hline 4 & Canonical flow & 1.5 & 0.138 & 0.050 & - \\
\hline 5 & Canonical flow & 2.0 & 0.138 & 0.085 & - \\
\hline 6 & Canonical flow & 1.1 & 0.354 & 0.010 & - \\
\hline 7 & Canonical flow & 1.1 & 0.569 & 0.006 & - \\
\hline 8 & Canonical flow & 1.1 & 0.785 & 0.002 & - \\
\hline 9 & Canonical flow & 2.0 & 0.354 & 0.061 & - \\
\hline 10 & Canonical flow & 2.0 & 0.569 & 0.048 & - \\
\hline 11 & Canonical flow & 2.0 & 0.785 & 0.035 & - \\
\hline 12 & Channel height $=4$ & 1.1 & 0.138 & 0.015 & Negligible \\
\hline 13 & Channel height $=16$ & 1.1 & 0.138 & 0.016 & Negligible \\
\hline 14 & Slightly diffuse, $c=0.5$ & 1.1 & 0.138 & 0.038 & - \\
\hline 15 & Moderately diffuse, $c=0.25$ & 1.1 & 0.138 & 0.028 & - \\
\hline 16 & Broadly diffuse, $c=0.125$ & 1.1 & 0.138 & 0.014 & . \\
\hline 17 & Sinusoid, $n=8, \bar{\epsilon}_{0}=0.05$ & 1.1 & 0.138 & 0.013 & Negligible \\
\hline 18 & Sinusoid, $n=8, \bar{\epsilon}_{0}=0.10$ & 1.1 & 0.138 & 0.012 & Negligible \\
\hline 19 & Sinusoid, $n=16, \bar{\epsilon}_{0}=0.05$ & 1.1 & 0.138 & 0.013 & Negligible \\
\hline 20 & Sinusoid, $n=16, \bar{\epsilon}_{0}=0.10$ & 1.1 & 0.138 & 0.014 & Negligible \\
\hline 21 & Ellipse, $\not R=0.5$ & 1.1 & 0.138 & 0.008 & -43 \\
\hline 22 & Ellipse, $R=2.0$ & 1.1 & 0.138 & 0.015 & 7 \\
\hline 23 & Ellipse, $R=0.5$ & 1.1 & 0.138 & 0.080 & -6 \\
\hline 24 & Ellipse, $R=2.0$ & 1.1 & 0.138 & 0.092 & 8 \\
\hline 25 & Late time reflected shock & 1.1 & 0.138 & 0.014 & 0 \\
\hline 26 & Intermediate time reflected shock & 1.1 & 0.138 & 0.026 & 86 \\
\hline 27 & Early time reflected shock & 1.1 & 0.138 & 0.035 & 150 \\
\hline 28 & Late time reflected shock & 1.2 & 0.138 & 0.027 & 13 \\
\hline 29 & Intermediate time reflected shock & 1.2 & 0.138 & 0.057 & 138 \\
\hline 30 & Early time reflected shock & 1.2 & 0.138 & 0.085 & 254 \\
\hline 31 & Late time reflected shock & 1.5 & 0.138 & 0.067 & 34 \\
\hline 32 & Intermediate time reflected shock & 1.5 & 0.138 & 0.132 & 164 \\
\hline 33 & Early time reflected shock & 1.5 & 0.138 & 0.222 & 344 \\
\hline 34 & Late time reflected shock & 2.0 & 0.138 & 0.157 & 88 \\
\hline 35 & Intermediate time reflected shock & 2.0 & 0.138 & 0.285 & 235 \\
\hline 36 & Streamwise pair, spacing $=6$ & 1.1 & $0.13 z$ & 0.014 & 0 \\
\hline 37 & Streamwise pair, spacing $=4$ & 1.1 & $0.13 ?$ & 0.018 & 29 \\
\hline 38 & Streamwise pair, spacing $=6$ & 1.2 & 0.138 & 0.032 & 33 \\
\hline 39 & Streamwise pair, spacing $=4$ & 1.2 & 0.138 & 0.040 & 67 \\
\hline 40 & Streamwise trio, spacing $=4$ & 1.1 & 0.138 & 0.015 & 7 \\
\hline 41 & Crosswise pair, spacing $=6$ & 1.1 & 0.138 & 0.015 & 7 \\
\hline 42 & Crosswise pair, spacing $=4$ & 1.1 & 0.138 & 0.015 & 7 \\
\hline 43 & Crosswise pair, spacing $=3$ & 1.1 & 0.138 & 0.018 & 29 \\
\hline 44 & Crosswise pair, spacing $=2.5$ & 1.1 & 0.138 & 0.018 & 29 \\
\hline 45 & Equilateral trio & 1.1 & 0.138 & 0.015 & 7 \\
\hline 46 & Equilateral trio & 1.2 & 0.138 & 0.025 & 4 \\
\hline 47 & Equilateral trio & 1.5 & 0.138 & 0.056 & 12 \\
\hline 48 & Equilateral trio & 2.0 & 0.138 & 0.136 & 60 \\
\hline
\end{tabular}

which can be rewritten as

$$
\frac{\mathrm{D} \omega}{\mathrm{D} t}=\frac{1}{\rho^{2}}(\nabla \rho \times \nabla p)+\cdots
$$

giving a rate of generation of vorticity proportional to the misalignment of the density and pressure gradients.

As the shock wave passes over the jet, vorticity is generated proportional to the misalignment of the density and pressure gradients. The discontinuity between light (jet) gas and heavy (ambient) gas produces a radially outward density gradient around the circumference of the jet. The shock wave provides an upstream-pointing pressure gradient. Where these two gradients are perpendicular, at the top and bottom of the jet, vorticity generation is maximum. Where the two gradients are parallel, at the upstream and downstream ends of the jet, vorticity generation is zero. At other circumferential locations, vorticity generation is intermediate.

Induced motions due to this vorticity initially cause the jet to roll up into a kidney-shaped structure. As time goes on, the vorticity coalesces and the structure evolves toward a vortex pair with finite core size.
Computations were performed for various combinations of shock strength and light/heavy gas density ratio. Figure 2 shows density contour plots for a computation of a $M=1.1$ shock and a light/heavy gas density ratio of $\bar{\rho}_{L} / \bar{\rho}_{H}=0.138$. The jet is located in the center of a channel of height eight times the jet radius. Each plot contains nine levels representing equally spaced values from near minimum to near maximum. The structure is essentially that proposed in Fig. 1, but with a thin filament connecting the vortex cores at the downstream end and trailing tails of low-vorticity material at the upstream end. The vortices are especially apparent in vorticity contour plots (Fig. 3). Figure 4 is a time history of the $f=0.05$ contour lengths for the flow of Fig. 2. The point of resolution loss is clearly seen, as is the asymptotic approach to exponential stretching prior to that point.

Lines 1-11 of Table 1 summarize the stretching rates for the single circular jet flows. In all cases, the channel height is eight times the jet radius. The stretching rate increases as either the density ratio is decreased or the shock strength is increased. This is a consequence of the dependence of vorticity generation on density or pressure gradient. Higher vorticity leads to increased induced motions and thus higher stretching rates. 


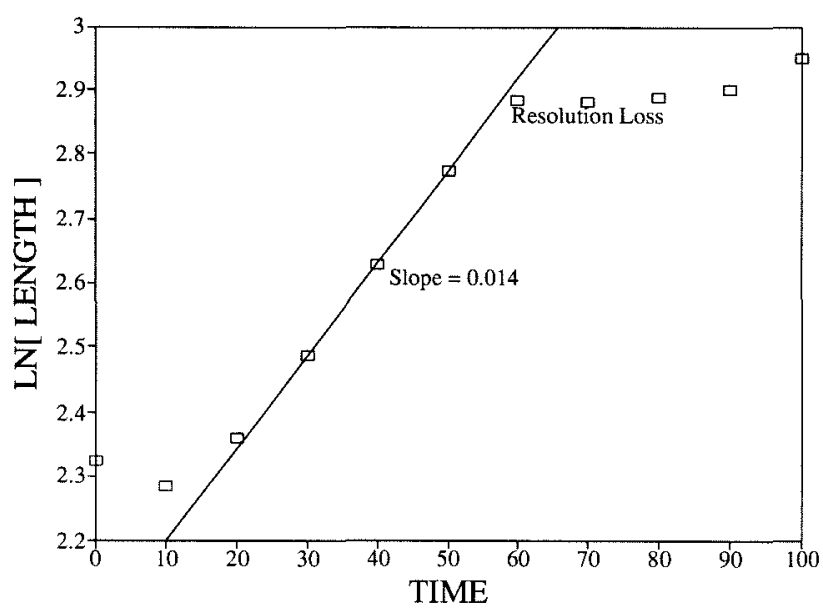

Fig. 4 Time history of $f=\mathbf{0 . 0 5}$ mass fraction contours for the flow of Fig. 2.

\section{Comparison with Experimental Data}

As a check on the accuracy of these stretching rates, one may compare against available experimental data. $\mathrm{Haas}^{2}$ and Haas and Sturtevant ${ }^{3}$ published shadowgraphs of weak shock waves in air impinging on cylinders of helium enclosed by weak nitrocellulose microfilms. Because shadowgraphs represent integrations along the optical path, different contour levels cannot be differentiated, and one must assume that the shadowgraph perimeter corresponds to some averaged value of $f$, say, $f_{\text {avg }}=0.5$. Therefore, stretching rates from the shadowgraphs should be compared to those of the $f=0.5$ contours in the computations.

Furthermore, it is necessary to use the entire time sequence of Refs. 2 and 3 to give enough data points to determine an asymptotic stretching rate, even though diffusion becomes important at late times. Thus the comparable stretching rate from the computations must also be the best straight-line value past the dropoff. This comparison will reflect the accuracy of the numerical diffusion. Digitization of the shadowgraphs of Refs. 2 and 3 gives the following:

$M=1.085, \bar{\rho}_{L} / \bar{\rho}_{H}=0.138$ :

$$
\begin{gathered}
\frac{\mathrm{D}}{\mathrm{D} \bar{t}}[\ln \bar{L}(f=0.5)]=0.026 \\
M=1.22, \bar{\rho}_{L} / \bar{\rho}_{H}=0.138: \\
\frac{\mathrm{D}}{\mathrm{D} \bar{t}}[\ln \bar{L}(f=0.5)]=0.080
\end{gathered}
$$

Stretching rates for the comparable single jet computations are as follows:

$$
M=1.1, \bar{\rho}_{L} / \bar{\rho}_{H}=0.138 \text { : }
$$

$$
\frac{\mathrm{D}}{\mathrm{D} \bar{t}}[\ln \bar{L}(f=0.5)]=0.033
$$

$$
M=1.2, \bar{\rho}_{L} / \bar{\rho}_{H}=0.138 \text { : }
$$

$$
\frac{\mathrm{D}}{\mathrm{D} \bar{t}}[\ln \bar{L}(f=0.5)]=0.073
$$

The good agreement reinforces the claim that the numerical diffusion, though inexact, is nevertheless a reasonable approximation to that in the real flow.

$\mathrm{Jacobs}^{7}$ studied interactions of weak shock waves with helium jets, using laser-induced fluorescence of a biacetyl tracer seeded into the helium. Experimental uncertainties were associated with calibrating the light variation across the laser sheet in any single image (a single time in a sequence) as well as among different images. Furthermore, the electronic camera used for data acquisition had a relatively poor signal-to-noise ratio at the lower intensity levels (corresponding to low mass fraction levels). For these reasons the highest confidence is associated with the median intensity level, again corresponding to $f=0.5$.

Jacobs performed three experiments: two $M=1.093$ runs, one near the jet exit (3.2 diameters downstream) and one far away (4.8 diameters downstream), and also a $M=1.22$ "far" run. Unknown variations in the camera angle in the $M=1.093$ "near" run preclude the use of those data, and an insufficient number of data points (time values) in the $M=1.22$ run preclude the use of those data. Digitization of images of the remaining case,,$^{13}$ the $M=1.093$ far run, gives a stretching rate of 0.034 , in agreement with the corresponding values from experiments of Refs. 2 and 3 and the present computations.

These comparisons verify the accuracy of the computations in predicting stretching rates for the corresponding real flows. Tabulated stretching rates in the remainder of the paper will refer to the $f=0.05$ mass fraction level appropriate to supersonic combustion.

\section{Effect of Channel Height}

The ratio of channel height to jet radius varied considerably among the experiments and the computations: 3.5 (Refs. 2 and 3) vs 17 (Jacobs) vs 8 (computations). The channel walls act as periodic planes of symmetry, forming an infinite array of image vortex pairs which contribute to the induced motion of every point in the flowfield. The good agreement in stretching rates despite wide variation in channel height suggests that induced motions from the image vortex pairs have insignificant effects on the stretching. To check this hypothesis, two additional channel heights, 4 and 16 , were computed for comparison against the baseline height of 8 . Contour plots are not shown because they are essentially unchanged. As shown in lines 12 and 13 of Table 1 , the stretching rates are essentially independent of channel height, as suggested by the comparison of experiments and computations.

\section{Effect of Interface Thickness}

The computations and Jacobs' experiments involved slices through jets at the same distance downstream of the jet exit. Slices through different downstream locations, having different mixing layer thicknesses, may affect the mixing. To investigate this computationally, it is convenient to use a hyperbolic-tangent distribution

$$
\frac{\bar{\rho}}{\bar{\rho}_{H}}=\frac{\bar{\rho}_{L}}{\bar{\rho}_{H}}+0.5\left(1-\frac{\bar{\rho}_{L}}{\bar{\rho}_{H}}\right)\left(1+\tanh \frac{c(\bar{r}-1)}{\Delta \bar{x}}\right)
$$

where $\Delta \bar{x}$ is the length of the side of each cell, $\bar{r}$ is the radial distance from the center of the jet, and the thickness is controlled by the single parameter $c$. This profile provides a smooth distribution of density from pure light gas at small $\bar{r}$ to pure heavy gas at large $\bar{r}$.

Lines 14-16 of Table 1 summarize the results for computations at various interface thicknesses. Contour plots are not shown because they are qualitatively similar to the canonical flow. As the interface thickness increases, the local density gradient decreases, and the stretching rate decreases. These stretching rates are not compared to stretching rates from the canonical flow because they are from different families of profiles.

This completes the discussion for the case of an isolated single circular jet. The remainder of the paper examines configurations that are more representative of actual technological applications. In all cases, the stretching rate will again refer to the $f=0.05$ mass fraction level of a jet initialized using the "mixing layer" profile. Families of single jet flows are considered first, followed by multiple jet flows. The single jet flows include perturbations about circular cross-sectional shape, noncircular cross sections, and reflected shock flows. The 
multiple jet flows include streamwise jet pairs, streamwise jet trios, crosswise jet pairs, and equilateral jet trios.

\section{Sinusoidal Perturbation to Cross-Sectional Shape}

In practice, circular jets are not always circumferentially smooth. The interaction of a shock wave with initial perturbations leads to the possibility of instabilities, which may contribute additional mixing across the interface.

Impulsive acceleration of a sinusoidally perturbed, plane interface was first studied by Richtmyer ${ }^{14}$ and Meshkov. ${ }^{15}$ The behavior depends on whether the shock propagates from heavy to light fluid, or vice versa. Consider a heavy/light interface. At early times, the perturbations exhibit negative amplitude growth, that is, they are stable. As time goes on, continued negative growth leads to phase reversal followed by unstable positive amplitude growth. A light/heavy interface exhibits positive (unstable) amplitude growth for all times, i.e., there is no stable precursor.

Now consider the more complicated case of a jet with small sinusoidal perturbations around its circumference. The initial configuration is given by

$$
\bar{r}(\theta)-1=\bar{\epsilon}_{0} \cos (n \theta)
$$

where 1 is the nominal radius of the jet, and $\bar{\epsilon}_{0} \ll 1$ and $n$ are the amplitude and wave number of the perturbation, respectively. The angle $\theta$ is measured counterclockwise from the downstream end.

Gross features of the flow should be similar to those of the unperturbed circular jet, because the mean shape is still circular. However, Richtmyer-Meshkov instabilities may be important where the mean density gradient is more or less parallel to the direction of the shock. The upstream (heavy/light) instabilities will initially experience damping. The downstream (light/heavy) instabilities will experience growth.

Figure 5 shows the case $M=1.1, \bar{\rho}_{L} / \bar{\rho}_{H}=0.138, n=16$, and $\bar{\epsilon}_{0}=0.1$, which is representative of this family of flows. The instabilities are significant at early times (first three frames). At the upstream interface, damping is coincident with development of the vortex pair, and the instabilities cease to exist as distinct phenomena before unstable growth can occur. At the downstream interface, the perturbations are unstable from the outset; note especially the growth of the first $(\theta=\pi / 8)$ instability. One would expect such growth to cause a corresponding increase in stretching rate compared to the single circular jet case.

Lines 17-20 of Table 1 show that the stretching rates for the sinusoidally perturbed circular jet flows are essentially unchanged from the circular jet case. Why is the expectation of increased stretching not realized? As a first approximation, one may describe the upstream and downstream instabilities by the equation for amplitude growth at a plane interface ${ }^{16}$ :

$$
\frac{1}{\bar{\epsilon}_{0}} \frac{\mathrm{d} \bar{\epsilon}}{\mathrm{d} t}=\frac{n \bar{\nu}_{2} A}{\psi}
$$

where $\bar{\epsilon}_{0}$ is the initial amplitude, $n$ the wave number, $\bar{v}_{2}$ is the velocity behind the shock, $A$ is the Atwood number $\left[ \pm\left(\bar{\rho}_{H}-\bar{\rho}_{L}\right) /\left(\bar{\rho}_{H}+\bar{\rho}_{L}\right)\right.$ at the upstream and downstream interfaces, respectivelyl, and $\psi$ is a growth reduction factor $(\psi>1)$ that depends on $|A|$ and interface thickness.

For the cases considered here, $\bar{v}_{2},|A|$, and $\psi$ are constant, so the growth rate is proportional to $n$ and $\epsilon_{0}$. Because their Atwood numbers are equal and opposite, the upstream and downstream instabilities have equal and opposite growth rates. Thus the overall stretching rate remains essentially constant, independent of the initial amplitude or wave number of the perturbation.

\section{Elliptical Jets}

Small perturbations to cross-sectional shape have little effect on the stretching. Is the same true for large variations in cross-sectional shape, such as in noncircular jets?
For an ellipse of aspect ratio $A \equiv b / a$, where $a$ and $b$ are the streamwise and crosswise semiaxes, $b$ replaces the radius of the circular jet in nondimensionalizing lengths and times, e.g., the nondimensional half-height of the ellipse is 1 . In general, the flows are qualitatively similar to the canonical flows except the streamwise dimensions are compressed or expanded depending on the initial aspect ratio.

Lines 21-24 of Table 1 summarize the stretching rates for several elliptical jet flows. At high Mach number, as the aspect ratio is increased, the streamwise dimension of the vortical structures is decreased, and the induced motions of the upstream and downstream interfaces are increased. This causes a slightly growing trend in the stretching rate. The same trend is observed at low Mach number, but there is significantly less stretching in the $A=0.5$ case. Examination of the $f=0.05$ contour would show it to have a small upstream protrusion, in contrast to the purely downstream protrusion of the inner (higher $f$ ) levels. This outlying fluid is so far upstream that it experiences almost no induced motion, and thus very little stretching. Stretching rates of higher mass fraction levels would probably be only slightly lower than in the $R=1.0$ and 2.0 cases. Overall, the elliptical jet stretching rates show a slight monotonic dependence on aspect ratio, and this dependence is greater at higher Mach number.

\section{Reflected Shock Interactions with a Vortex Pair}

Up to this point, the shock wave was always assumed to leave the domain after interacting with the jet. But real flows may involve closed domains, with associated reflected shocks.

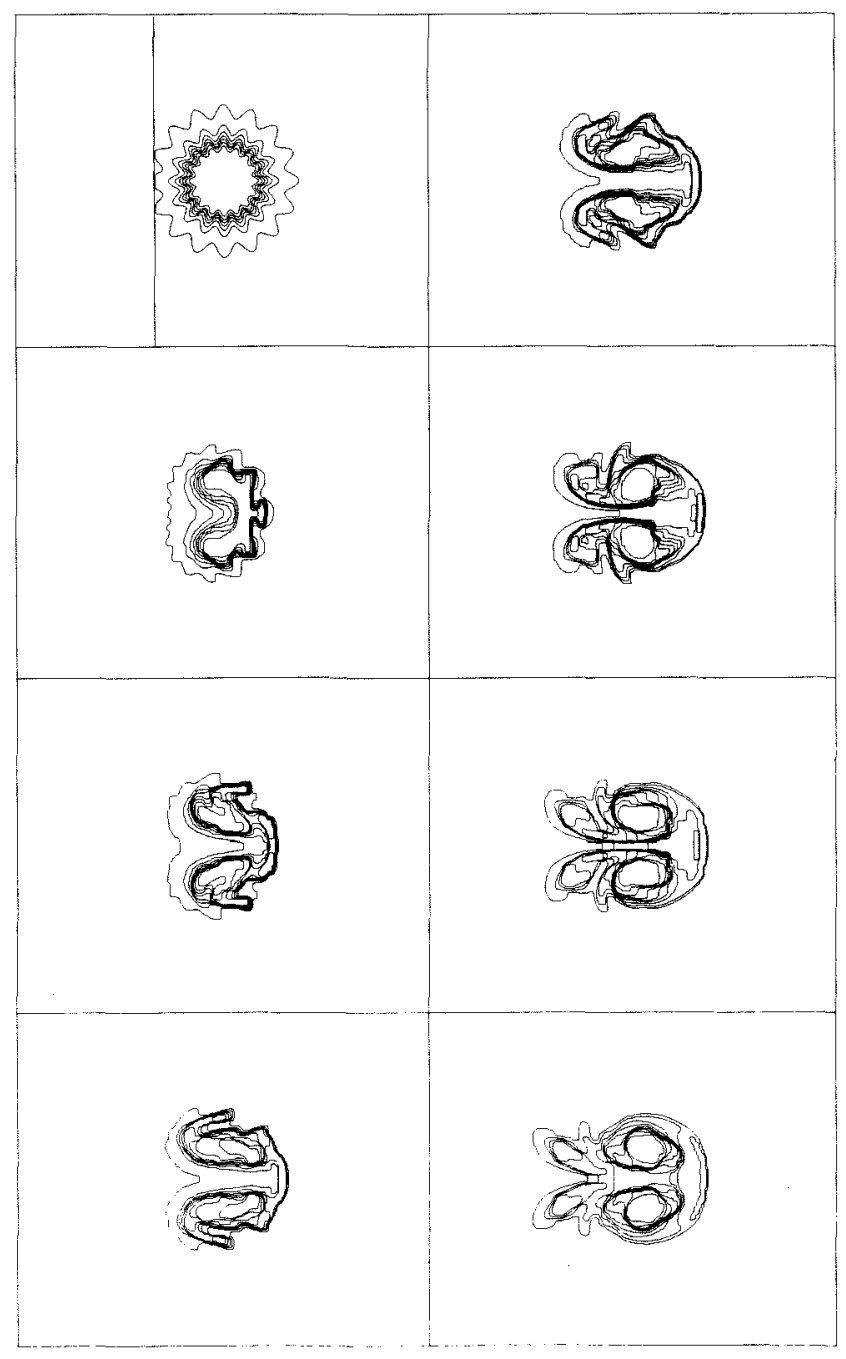

Fig. 5 Density contours at $\bar{t}=0-70$ for a sinusoidal jet with $M=1.1, \bar{\rho}_{L} / \bar{\rho}_{H}=0.138, n=16$, and $\epsilon_{0}=0.1$. 
For example, consider a $M=1.1, \bar{\rho}_{L} / \bar{\rho}_{H}=0.138$ circular jet (initially the same as Fig. 2). Figure 6 shows the late time flow when the reflected shock is about to pass over the vortical structure created by the incident shock. Consider the upper half-plane. The vortex core is an approximately circular region of light gas, surrounded by heavy gas, similar to the initial jet but having a strong initial counterclockwise vorticity. In the absence of this vorticity, the core would behave like the initial jet: vorticity deposited in its outer half would develop into a clockwise outer vortex, vorticity deposited in its inner half would develop into a counterclockwise inner vortex, and these vortices would be approximately the same size.

However, the core's strong initial counterclockwise vorticity suppresses the outer half's development and enhances the inner half's development. The result is a nonsymmetrical vortex pair, consisting of a small, clockwise outer vortex rotating counterclockwise about a large, counterclockwise inner vortex. This partitioning of the vortex core into another vortex pair significantly increases the stretching beyond the case with no reflected shock.

Computations were also performed for intermediate time and early time reflected shocks, where the reflected shock passes over a developing vortical structure that has not yet stabilized into a vortex pair. The reflected shock generates additional vorticity of both positive and negative sense, and it is the preferential coalescence of same-sense vorticity that determines the relative sizes of the inner and outer vortices. The earlier the reflected shock hits, the less stable is the

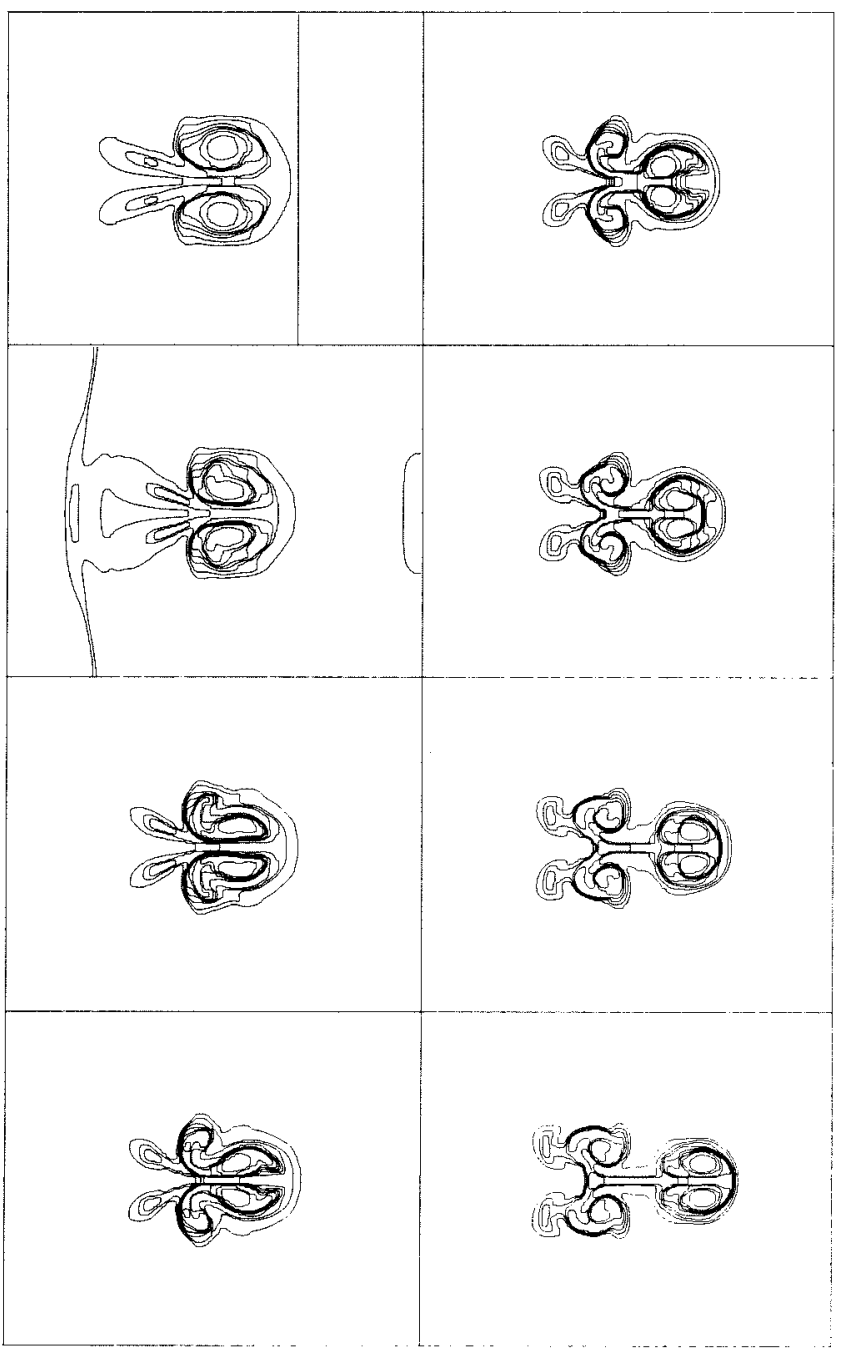

Fig. 6 Density contours at $\bar{t}=0$-70 for a late time reflected shock with $M=1.1$ and $\bar{\rho}_{L} / \bar{\rho}_{H}=0.138$.

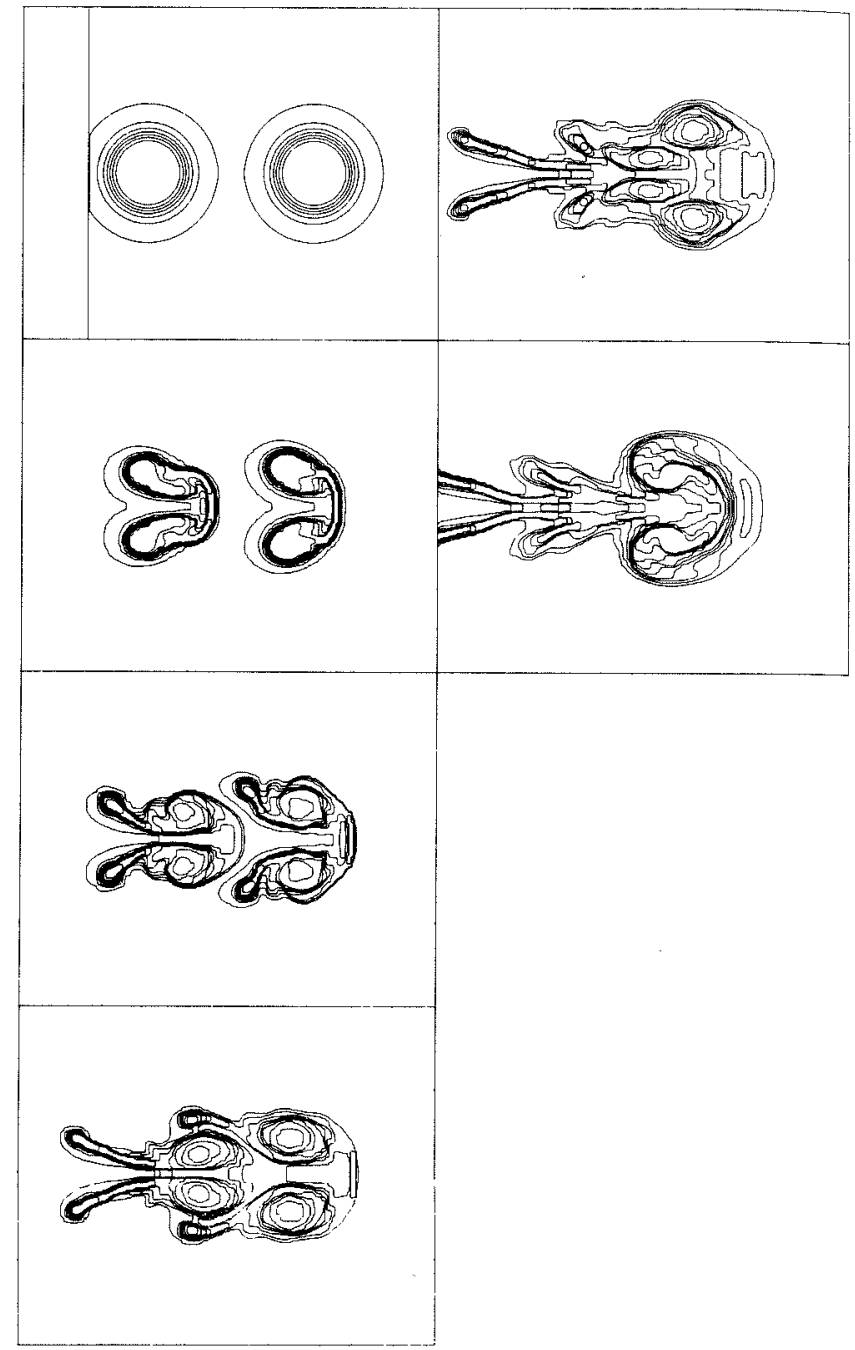

Fig. 7 Density contours at $\bar{t}=0-70$ for a streamwise jet pair with $M=1.1, \bar{\rho}_{L} / \bar{\rho}_{H}=0.138$, and spacing $=4$.

original vortex pair, and the weaker is its ability to append the additional vorticity due to the reflected shock. Each half of the original vortex pair is again split into a strong inner vortex and a weak outer vortex, but these vortices become more nearly equal in size the earlier the reflected shock hits. The more equitable partitioning of the vortex core also results in greater stretching.

Lines 25-35 of Table 1 summarize the stretching rates for the reflected shock flows. The expectation of greatly increased stretching is confirmed by the computations. The earlier the reflected shock hits, the greater the increase.

\section{Streamwise Jet Pair}

Only single jets have been considered so far, but real technological applications will probably involve multiple jets. The simplest configuration is a streamwise pair of jets. A family of such cases is computed, using superposition of single jet profiles to initialize the domain.

If the jets are sufficiently far apart, they form two noninteracting vortex pairs, each behaving as if it were the only one in the field. If the initial spacing is decreased or the shock strength is increased, the two vortex pairs will interact, in a manner that can be predicted from simple induced motion considerations. Consider two identical vortex pairs, one upstream of the other. Each vortex will experience an induced motion component from every other vortex, inversely proportional to their separation. The net motions are found by vector summation. Overall, the downstream pair will move apart, reducing its downstream velocity, causing it to decelerate. The 


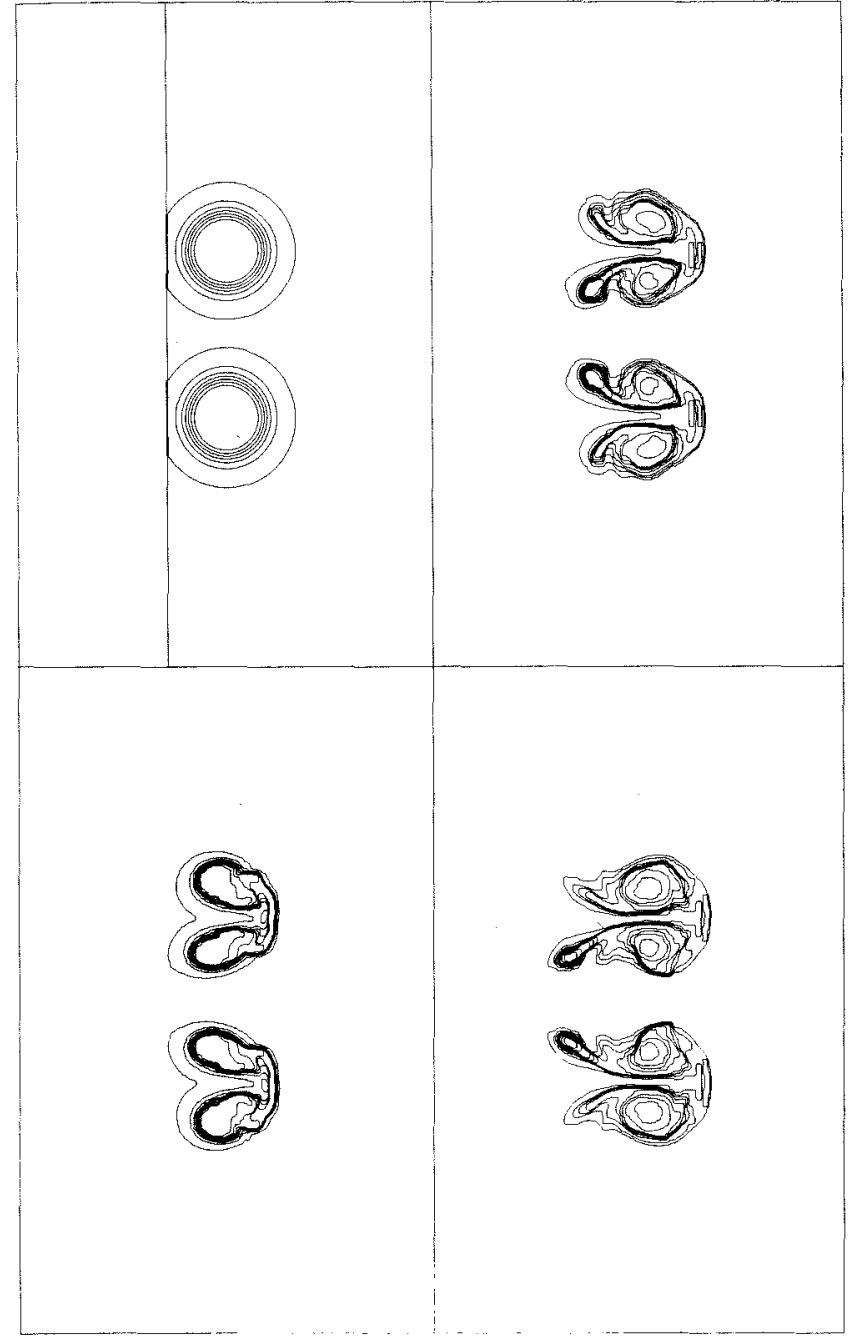

Fig. 8 Density contours at $\bar{t}=0-90$ for a crosswise jet pair with $M=1.1, \bar{\rho}_{L} / \bar{\rho}_{H}=0.138$, and spacing $=4$.

upstream pair will move together, increasing its downstream velocity, causing it to accelerate. Thus, the upstream vortex pair will be entrained into the downstream one. This will cause the tails of the downstream vortex pair to be pulled up and around the outer edge of the upstream vortex cores. Both the entrainment and the tail stretching should lead to increased stretching beyond the single circular jet case.

These expectations are verified by the computations. For example, Fig. 7 shows the case $M=1.1, \bar{\rho}_{L} / \bar{\rho}_{H}=0.138$, and spacing $=4$. At intermediate times, notice the decrease in crosswise spacing of the upstream vortex pair as it accelerates toward the downstream pair. The tails of the downstream structure are pulled upstream, around the outer edges of the upstream vortex cores. At late time, the entrainment becomes so strong that the upstream vortex pair merges into the downstream pair, and the overall structure reorganizes to form a single stable vortex pair.

Lines $36-39$ of Table 1 summarize the stretching rates for the streamwise jet pair flows. The closer the jets are initially, the greater the stretching, and the stronger the shock, the greater the increase.

\section{Streamwise Jet Trio}

Another configuration of interest is that of a trio of streamwise jets. This configuration is an extension of the streamwise jet pair, and much of the same phenomena are observed. However, the middle vortex pair is less likely to be entrained into the downstream vortex pair, because of the retarding influence of the upstream vortex pair. Therefore, the stretch- ing rate (line 40 , Table 1 ) is less than that for a streamwise jet pair with the same spacing.

\section{Crosswise Jet Pair}

Besides streamwise jet configurations, crosswise configurations are also technologically important. Consider a pair of identical circular jets located symmetrically about the centerline of a channel. Note that a jet spacing of 8 radii would correspond exactly to the canonical single circular jet case, because the array would be perfectly periodic.

Figure 8 shows the case $M=1.1, \bar{\rho}_{L} / \bar{\rho}_{H}=0.138$, and jet spacing $=4$. Even at early times, the inner portions of the vortical structures induce motions in each other that lead to pronounced asymmetry. This causes them to pinch off larger tails than the corresponding single circular jet. Since more of the fluid is in the tails, the vortex cores must be correspondingly smaller. Conversely, the outer tails are smaller and the outer vortex cores are larger than in the single circular jet. The difference in vortex core sizes results in the small inner vortex core being rotated around the larger outer vortex core at $\bar{t}>50$. As a consequence, the stretching rate is greater than the corresponding single jet.

Lines 42-44 of Table 1 summarize the stretching rates for the crosswise jet pair flows. In all cases, the channel height is 16. The stretching rates are less than those of the streamwise jet pairs at the same Mach number and spacing.

\section{Equilateral Jet Trio}

Finally, consider three jets in an equilateral trio formation, with center-to-center spacing of 3 radii and upstream-facing vertex. For example, the case of $M=1.1$ and $\bar{\rho}_{L} / \bar{\rho}_{H}=0.138$ is shown in Fig. 9. The flow shows both the entrainment charac-

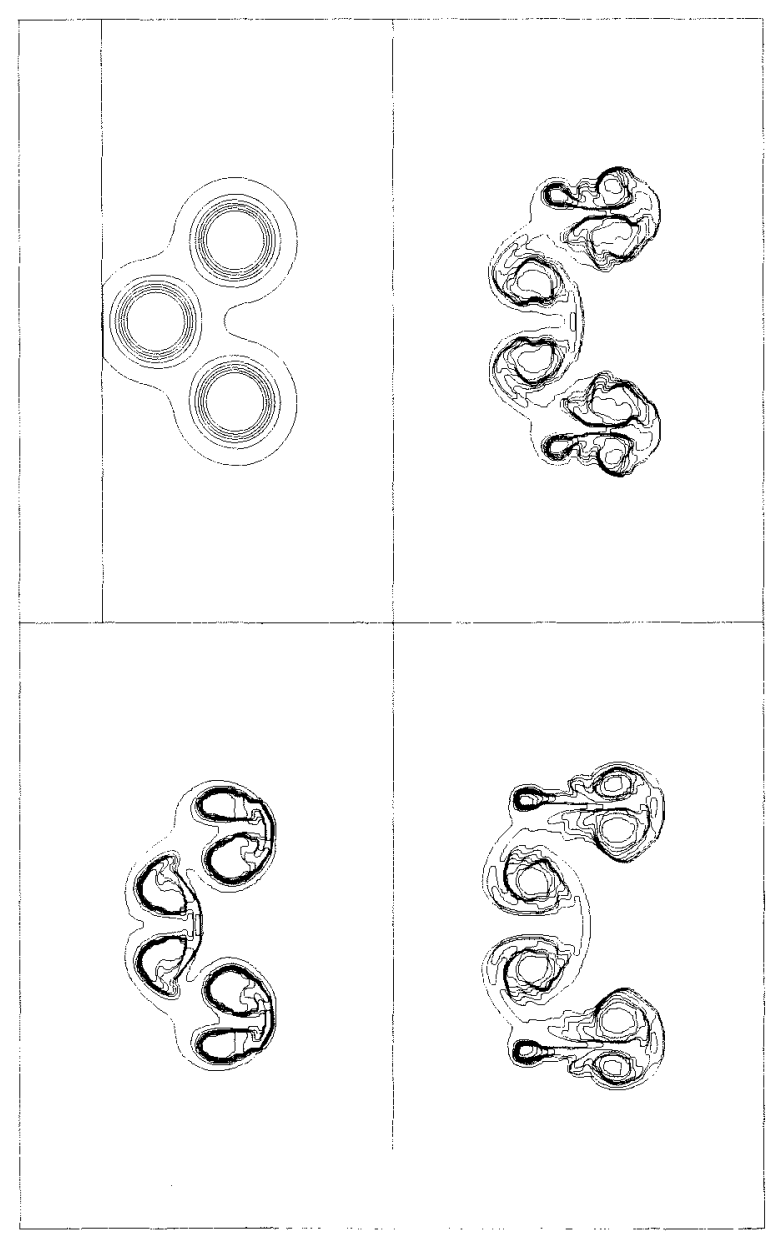

Fig. 9 Density contours at $\bar{t}=0-90$ for a equilateral jet trio with $M=1.1, \bar{\rho}_{L} / \bar{\rho}_{H}=0.138$, and spacing $=4$. 
teristic of the streamwise jet pairs and the asymmetric development characteristic of the crosswise jet pairs.

Lines $45-48$ of Table 1 summarize the computed stretching rates for the equilateral jet trios. The stretching is increased from that of a single jet, and the greater the Mach number, the greater the increase in stretching.

\section{Summary of the Two-Dimensional, Unsteady Stretching Studies}

The stretching rate can be increased by variation of fluid mechanical parameters. The stronger the incident shock or the initial density difference, the larger the stretching rate. Also, the sharper the initial interface, the greater the stretching. Geometrical variations are relatively ineffective in increasing the stretching rate for single jets. Sinusoidal perturbations show no improvement, because the effects at the upstream and downstream edges cancel. Elongation normal to the flow slightly increases the stretching, whereas elongation parallel to the flow slightly decreases the stretching. The stretching is greatly increased if a reflected shock hits the vortex pair formed by the incident shock. The sooner the reflected shock hits, the better. Of the multiple jet configurations, streamwise jet pairs are the most effective, followed by crosswise jet pairs. Streamwise jet trios and equilateral triangle configurations also slightly increase the stretching rate.

\section{Applications to Supersonic Combustion}

For SCRAMJET applications, one or more jets of fuel must be injected into and mixed with a coflowing supersonic air stream. In Refs. 1, 6, 17, and 18 it is argued that this three-dimensional, steady interaction of a light gas jet with an oblique shock wave is analogous to the two-dimensional, unsteady canonical problem. Spatial passage of the oblique shock in the three-dimensional flow corresponds to temporal passage of a normal shock in the two-dimensional flow.

There are numerous ways of generating vorticity in three-dimensional, steady flows, depending on the particular design of the injector. However, the two-dimensional, unsteady flows of the present work directly correspond only to three-dimensional, steady flows in which the predominant mechanism of vorticity generation is baroclinic. Waitz et al. ${ }^{19}$ compared injector flows involving two competing vorticity generation mechanisms and found baroclinic vorticity generation to be the most effective in lifting the jet away from the combustor wall. In an actual SCRAMJET, large liftoff is necessary to avoid excessive wall heating. This suggests that baroclinic generation of vorticity is indeed the preferred mechanism for three-dimensional, steady flows.

The necessity for a large liftoff (three-dimensional) corresponds to a large downstream velocity (two-dimensional). Since it is the vorticity that induces the motion of the vortex pair, and greater vorticity is produced by greater misalignment of the initial density and pressure gradients, this suggests a shape with much of its perimeter perpendicular to the motion of the shock wave, e.g., a tall and narrow injector (three-dimensional) or a jet of low aspect ratio (two-dimensional). This is qualitatively similar to the two-dimensional ellipse of low aspect ratio, which showed only a weak decrease in mixing for a substantial decrease in aspect ratio.

Next, given the requirement for injection of a fixed amount of fuel into the domain, how should the injectors be sized? Multiple arrays of smaller injectors are preferable to a single, larger injector. Furthermore, the injectors should be arranged in groups of closely spaced pairs rather than uniformly spaced.

Finally, the use of multiple shocks, as early as possible following the passage of the primary shock, is strongly recommended, provided that the additional losses associated with multiple shocks are acceptable. These are competing design considerations which must be optimized on a case-by-case basis. In the three-dimensional case, this could take the form of a reflected shock from the upper combustor wall, or a secondary shock located downstream of the primary shock.

\section{Acknowledgments}

This work was supported by the Air Force Office of Scientific Research, under the supervision of Julian Tishkoff, through Contract F49620-86-C-0113 and Grant AFOSR-900188. It was also supported by the National Science Foundation through a Cray supercomputer grant at the San Diego Supercomputer Center. Joseph Yang was supported by the Office of Naval Research through an ONR Graduate Fellowship. The authors would like to thank Elaine S. Oran, of the Naval Research Laboratory, for providing the algorithm used in the numerical simulations.

\section{References}

${ }^{1}$ Marble, F. E., private communication, California Inst. of Technology, Pasadena, CA, Jan. 1985.

${ }^{2}$ Haas, J.-F., "Interaction of Weak Shock Waves with Cylindrical and Spherical Gas Inhomogeneities," Ph.D. Thesis, Dept. of Aeronautics, California Inst. of Technology, Pasadena, CA, June 1983.

${ }^{3}$ Haas, J.-F., and Sturtevant, B., "Interaction of Weak Shock Waves with Cylindrical and Spherical Gas Inhomogeneities," Journal of Fluid Mechanics, Vol. 181, 1987, pp. 41-76.

${ }^{4}$ Rudinger, G., and Somers, L. M., "Behaviour of Small Regions of Different Gases Carried in Accelerated Gas Flows," Journal of Fluid Mechanics, Vol. 7, 1960, pp. 161-176.

5Picone, J. M., and Boris, J. P., "Vorticity Generation by Shock Propagation Through Bubbles in a Gas," Journal of Fluid Mechanics, Vol. 189, 1988, pp. 23-51.

${ }^{6}$ Hendricks, G. J., and Marble, F. E., "Shock Enhancement of Supersonic Combustion Processes," preliminary draft of a manuscript in preparation, Dept. of Mechanical Engineering, California Inst. of Technology, Pasadena, CA, 1991.

7Jacobs, J. W., "Shock Induced Mixing of a Light Gas Cylinder," Journal of Fluid Mechanics, Vol. 234, 1992, pp. 629-649.

${ }^{8}$ Yang, J., Kubota, T., and Zukoski, E. E., "An Analytical and Computational Investigation of Shock-Induced Vortical Flows," AIAA Paper 92-0316, Jan. 1992.

${ }^{9}$ Boris, J. P., Landsberg, A. M., Oran, E. S., and Gardner, J. H., "LCPFCT - A Flux Corrected Transport Algorithm for Solving Generalized Continuity Equations," Naval Research Lab. Memorandum Report, Washington, DC, 1993.

${ }^{10}$ Boris, J. P., "SHAS2D-A Fully Compressible Hydrodynamics Code in Two Dimensions," Naval Research Laboratory Memo NRLMR-2542, Naval Research Laboratory, Washington, DC, 1977.

${ }^{11}$ Batchelor, G. K., "The Effect of Homogeneous Turbulence on Material Lines and Surfaces," Proceedings of the Royal Society of London, Vol. 213A, 1952, pp. 349-366.

${ }^{12}$ Leonard, A., private communication, California Inst. of Technology, Pasadena, CA, Jan. 1991.

${ }^{13}$ Jacobs, J. W., unpublished data, California Inst. of Technology, Pasadena, CA, Jan. 1991

${ }^{14}$ Richtmyer, R. D., "Taylor Instability in Shock Acceleration of Compressible Fluids," Communications in Pure and Applied Mathematics, Vol. XIII, 1960, pp. 291-319.

${ }^{15}$ Meshkov, T. T., "Instability of a Shock Wave Accelerated Interface Between Two Gases," NASA TT F-13074, 1970.

${ }^{16}$ Brouillette, M., "On the Interaction of Shock Waves with Contact Surfaces Between Gases of Different Densities," Ph.D. Thesis, Dept of Aeronautics, California Inst. of Technology, Pasadena, CA, May 1989.

${ }^{17}$ Marble, F. E., Hendricks, G. J., and Zukoski, E. E., "Progress toward Shock Enhancement of Supersonic Combustion Processes," AIAA Paper 87-1880, June 1987

${ }^{18}$ Marble, F. E., Zukoski, E. E., Jacobs, J. W., Hendricks, G. J., and Waitz, I. A., "Shock Enhancement and Control of Hypersonic Mixing and Combustion," AIAA Paper 90-1981, July 1990.

${ }^{19}$ Waitz, I. A., Marble, F. E., and Zukoski, E. E., "An Investigation of a Contoured Wall Injector for Hypervelocity Mixing Augmentation," AIAA Paper 91-2265, June 1991. 